\title{
Oscillatory thresholds and foveal and macular thickness using coherence tomography
}

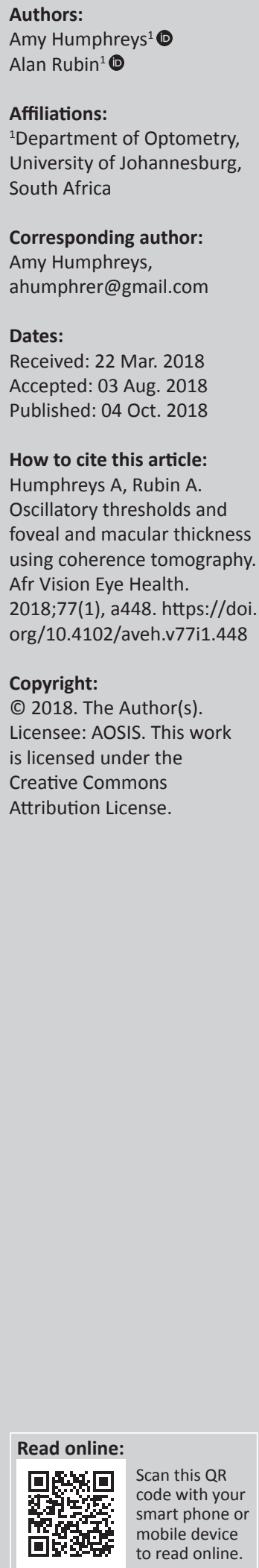

Background: Technology is evolving and advancing rapidly as is evident in the ophthalmic and optometric fields with the development of new equipment and software programs that assist in the examination of patients and diagnoses of ocular diseases. The new vision psychophysical software for measurements of oscillatory sensitivity (OS) is a recently developed program that can be used to assess visual ability at the fovea by establishing oscillatory thresholds (OT) and it is therefore necessary to assess the reliability and repeatability of this new software.

Aim: The goal of this study was to assess the reliability and repeatability of the new vision psychophysical software for measuring OS with special focus on the macular and foveal area.

Setting: This study took place at the Department of Optometry at the University of Johannesburg between 2016 and 2017.

Methods: To measure OS, an ascending threshold method was used where a simple target was presented, with increasing oscillations until perception of movement occurred. Healthy participants $(N=37)$, with a mean age of $22.43 \pm 2.57$ years, were asked to indicate when the high contrast circular $(2 \mathrm{~mm})$ target began oscillating on a stationary black background. The target was used at nine points in the macular region to measure OT. Each macular map was measured twice per participant and only the right eyes were involved. Retinal thicknesses were also evaluated using the spectral-domain iVue 100 optical coherence tomography (OCT) from Optovue. Macular thickness and OS (along with OT) measurements were assessed using various graphical and statistical methods to establish test-retest reliability and repeatability.

Results: The study focused on OS and retinal thickness measurements of the macular and foveal regions. For retinal thickness measurements, repeatability and reliability were good, data was normally distributed and agreement between test-retest measurements was high, while OS measurements had good repeatability and reliability, were not normally distributed but had good agreement between test-retest measurements. With healthy participants, no correlations between OCT and OS measurements were found $(r=-0.14, p=0.49$ and $r=0.03$, $p=0.89$ for test and retest samples).

Conclusion: The results for both procedures suggest that there is good reliability and repeatability within the macular region. Both methods can be used with confidence clinically and in future studies.

\section{Introduction}

Many recent advances have been made in ophthalmic and optometric practice with regards to diagnosing ocular diseases. Although these advances have been beneficial for both the examiner and patient, some of the advances in technology have resulted in expensive instrumentation and sometimes time-consuming tests for both examiners and patients. Therefore, new vision psychophysical software (preliminarily named Vision Psychophysical Software version 1 or VPS1 and developed by Professor Alan Rubin of the Department of Optometry, University of Johannesburg) concentrating on oscillatory sensitivity (OS) was investigated as a means to quickly and accurately assess visual ability at the fovea and surrounding retina.

Oscillatory thresholds (OT) and OS are fairly new concepts whereby participants are asked to indicate when they perceive a target to be oscillating. Oscillation is the process whereby a target moves to and fro (with a type of vibration). ${ }^{1}$ Because of OS being subjectively specific, visual perception studies often need both subjective and objective means of investigating vision. ${ }^{2,3}$ Along with visual perception being individually specific, many theories, such as Gestalt principles, exist that could possibly complicate conclusions on visual perception, ${ }^{3,4}$ but these will not be elaborated on in this article. 
Although many studies on motion perception with a variety of stimuli have previously been conducted, no studies have been completed using either the new vision psychophysical software (VPS1) or a simple single oscillating target, which was chosen to avoid any user confusion. In addition, previous studies have used more complicated targets, including sinusoidal gratings, random-dot cinematograms, drifting Gabor patches ${ }^{5,6,7}$ or complicated arrangements of dots moving in different directions. ${ }^{8}$ Therefore, it was necessary to assess repeatability and reliability for a simple, single oscillating target.

Optical coherence tomography (OCT) is a quick and noninvasive method that produces high-resolution cross sections of biological tissues such as the retinal structures. ${ }^{9,10,11}$ An optical coherence tomographer is able to convert a threedimensional image into a two-dimensional scan. ${ }^{12,13}$ Several studies have been conducted regarding macular thickness measurements and repeatability, with few having used the iVue 100 OCT as the instrument for measuring them. Studies in South Africa conducted by Mashige and Oduntan ${ }^{14}$ and Murugan et al. ${ }^{15}$ used the iVue 100 OCT to assess average macular thicknesses in the African population (268.75 \pm 15.04 $\mu \mathrm{m})$ and average macular thicknesses in African and Indian myopic patients ( $238 \pm 28 \mu \mathrm{m}$ and $243 \pm 18 \mu \mathrm{m}$, respectively). Although both studies ${ }^{14,15}$ used the iVue 100 OCT instrument to obtain macular thickness measurements, these studies concentrated on one type of refractive error and/or subpopulation. Other studies, using OCT instruments such as the Cirrus, Spectralis, Stratus and RTVue, found average macular thicknesses ranging from $175.71 \mu \mathrm{m} \pm 18$ to $289 \pm$ $16 \mu \mathrm{m} \cdot{ }^{16,17,18,19}$ Differences in macular thicknesses may be a result of many factors including age, ethnicity, gender ${ }^{20}$ and ocular factors such as refractive error and axial lengths. ${ }^{21}$ It is proposed that differences in retinal thickness measurements may also be attributed to varying inner and outer boundaries set for measuring retinal thicknesses for different OCT instruments. The aim of this study was to investigate test and retest reliability and repeatability of the new software procedure with special attention to finding very preliminary data for OT as well as investigating reliability of macular thickness measurements using the iVue 100 OCT instrument. Possible correlation between the two procedures was also explored. For this study, special attention was directed towards the macular and foveal regions for both OT and iVue 100 OCT measurements.

\section{Methodology \\ Participant selection}

Participants were selected using convenience sampling and were comprised of mainly students from the Department of Optometry at the University of Johannesburg. The study was approved (HDC-01-02-2016 and REC-01-34-2016) by the relevant structures of the University of Johannesburg. All participants were provided with information concerning the aims and methods of the study as well as possible risks and benefits. Healthy participants, aged between 18 and 30 years, within the refractive error criteria to be discussed, were included. Participants provided consent and completed a biographical questionnaire regarding ocular and general health. A total of 37 participants took part, of which 26 participants were included (11 participants had measurements that were not reliable and therefore were excluded) in the focused macular and foveal study, which is the emphasis of this article. The 26 participants were between 18 and 30 years of age with a mean age of $22.77 \pm 2.86$ years. The median age and quartile deviation $(=1 / 2$ interquartile range) was $22 \pm 2$ years. The sample had an equal distribution of men $(n=13)$ and women $(n=13)$, who were mainly of Caucasian $(46.15 \%)$ and African (30.77\%) descent. Participants were excluded if they had any history of ocular injuries or were using chronic medications. Although head trauma was a possible contraindication for participation, in this study, one participant who underwent head surgery was allowed to continue as the participant was healthy and showed no signs of ocular and/or visual deviations. Additionally, this participant was specifically included to investigate whether or not OS is affected by previous head trauma.

\section{Preliminary tests}

Preliminary tests were conducted to ensure participants satisfied the criteria set for this study. Visual acuities (VA) at distance $(6 \mathrm{~m})$ and near $(40 \mathrm{~cm})$ were tested using a Snellen distance chart and Lighthouse near chart. All participants needed to have VA of $6 / 6$ at distance and near with or without habitual correction. Those who had 6/6 unaided VA at near were asked not to use their refractive compensation during OT tests. Three autorefractor readings were obtained per eye, using the Topcon KR-1W Wave-Front Analyzer, to ensure participants satisfied the refractive constraints of no more than $\pm 2.00 \mathrm{D}$ for the sphere $\left(F_{\mathrm{s}}\right)$ and $(0 ;-2) \mathrm{D}$ for the cylinder $\left(F_{c}\right)$. Binocularity screening was completed using the cover test. Ocular health was assessed using a slitlamp and Canon CR-2 Plus Digital Retinal Camera for the anterior and posterior ocular segments, respectively. Where necessary, ophthalmoscopy was performed. Intraocular pressures were also measured using a Keeler Pulsair Easy Eye Tonometer. Once participants underwent the preliminary tests and were eligible for the study based on the inclusion criteria, the iVue 100 OCT measurements and OT tests were performed. Participants who presented with high refractive errors, poor distance and near VA, ocular diseases and/or chronic conditions (such as diabetes and high blood pressure) were excluded.

\section{iVue 100 ocular coherence tomography measurements}

For this study, the Optovue iVue 100 OCT instrument was used to measure the retinal thickness. Four scans, which included two retinal 3D scans and two ganglion cell complex scans, were completed for the right eye of each participant. Each type of scan was performed twice (test and retest) on each participant's right eye, resulting in 26 test and 26 retest measurements for each type of scan. 


\section{Oscillatory threshold measurements}

To investigate the macular OT, macular field tests were conducted on the right eye of each participant $(n=26)$. All participants were seated $50 \mathrm{~cm}$ from a computer monitor (Dell $69 \mathrm{~cm}$ with a refresh rate of $59 \mathrm{~Hz}$ and power rating of $100 \mathrm{~V}-240 \mathrm{~V})$ with their left eyes occluded. Participants were instructed to fixate on the central red fixation target for the duration of the tests. Along with the central red fixation target, a white circular target $(2 \mathrm{~mm})$ would appear within the visual field being evaluated (macular OS field tests [MF 9]) and participants were asked to click the Stop button as soon as they perceived that the white target was oscillating. When necessary, short breaks were taken. For the macular OS field tests (MF 9), nine points were used in a fixed grid (Figure 1) on the computer screen, subtending approximately 4 degrees of the visual field.

Five specific retinal regions and five points of the nine were chosen to further investigate retinal thicknesses and OT because of the ability to correlate specific retinal sections with the OT points tested. For OT, Points 2, 4, 5, 6 and 8 were chosen, which also correlates to inferior inner macular (IIM), temporal inner macular (TIM), central subfield/fovea (CSF), nasal inner macular (NIM) and superior inner macular (SIM), respectively (see Figures 1 and 2 , respectively).

For the macular field tests (MF 9), pseudo-3D maps were produced (Figures 3-6). According to the colour bar for the OT thresholds in micrometres (on the left side of the maps), warmer colours such as red indicate poorer OS (increased OT because of larger oscillatory displacements) while cooler colours such as blue indicate better OS (decreased OT because of smaller oscillatory displacement). Therefore, if thresholds in micrometres were larger, greater oscillation was needed before motion was perceived and OS was weaker. It should be noted that although the thresholds were measured in micrometres $(\mu \mathrm{m})$, the $x$ - and $y$-coordinates of the pseudo-3D maps were constructed using millimetres $(\mathrm{mm})$.

\section{Statistical analysis}

Statistical analysis was conducted on measurements of both retinal thickness and OT including tests for normality, hypothesis testing, Bland-Altman plots to assess agreement between test and retest measurements and correlation between retinal thickness measurements and OT. The statistics used for the normality tests consisted of Kolmogorov-Smirnov, Lilliefors and Shapiro-Wilk probabilities. Kolmogorov-Smirnov is based on the maximum difference between sample distributions and hypothesised distribution. It is denoted with a $D$ statistic that if significant means that the hypothesis that the distribution of interest is normal should be rejected. ${ }^{22,23}$ Lilliefors is used in conjunction with Kolmogorov-Smirnov

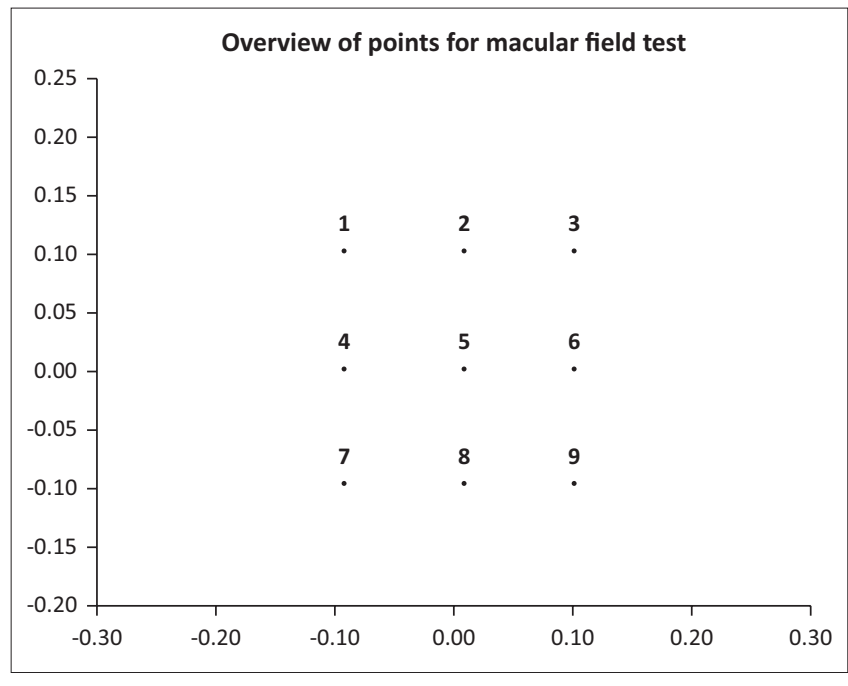

FIGURE 1: Schematic diagram of macular oscillatory sensitivity field test with the nine points. The foveal point $(0.0 ; 0.0)$ is portrayed by Point 5 . As can be seen by the grid, Points 1 to 9 have the following coordinates: $(-0.10 ; 0.10),(0 ; 0.10)$, $(0.10 ; 0.10),(-0.10 ; 0.0),(0.0 ; 0.0),(0.1 ; 0.0),(-0.10 ;-0.10),(0.0 ;-0.10)$ and $(0.10 ;-0.10)$, respectively.

and supports or rejects the findings of KolmogorovSmirnov tests. ${ }^{24}$ Shapiro-Wilk is often preferred over the Kolmogorov-Smirnov because it requires only one test to make a conclusion on normality whereas the KolmogorovSmirnov often needs other tests to assist. Agreement between test and retest measurements for both retinal thickness and OT were investigated using Bland-Altman plots. In the Bland-Altman plots, means versus the differences of the test and retest measurements are indicated using dots for retinal thickness and OT separately in Figure 7. Correlation between retinal thickness measurements and OT was investigated using scatterplots and Pearson's correlation coefficients (Figure 8).

\section{Ethical consideration}

The study conformed to the tenets of the 2013 Declaration of Helsinki and was approved by the Higher Degrees and Ethics Committees (HDC-01-02-2016 and REC-01-34-2016, respectively) of the Faculty of Health Sciences, University of Johannesburg, South Africa.

\section{Results}

\section{iVue 100 optical coherence tomography measurements}

The mean retinal thickness and standard deviation (SD) for the nine Early Treatment Diabetic Retinopathy Study (ETDRS) regions for the 26 right eyes are shown in Figure 2. For each region, the test means and SD can be seen above while the retest means and SD are found below the test thicknesses in square brackets. The mean test and retest retinal thickness measurements were fairly similar in the nine ETDRS regions. The maximum and minimum regional retinal thickness differences were $5 \mu \mathrm{m}$ (inferior outer macular [IOM]) and $0.1 \mu \mathrm{m}$ (temporal outer macular [TOM]), respectively. 


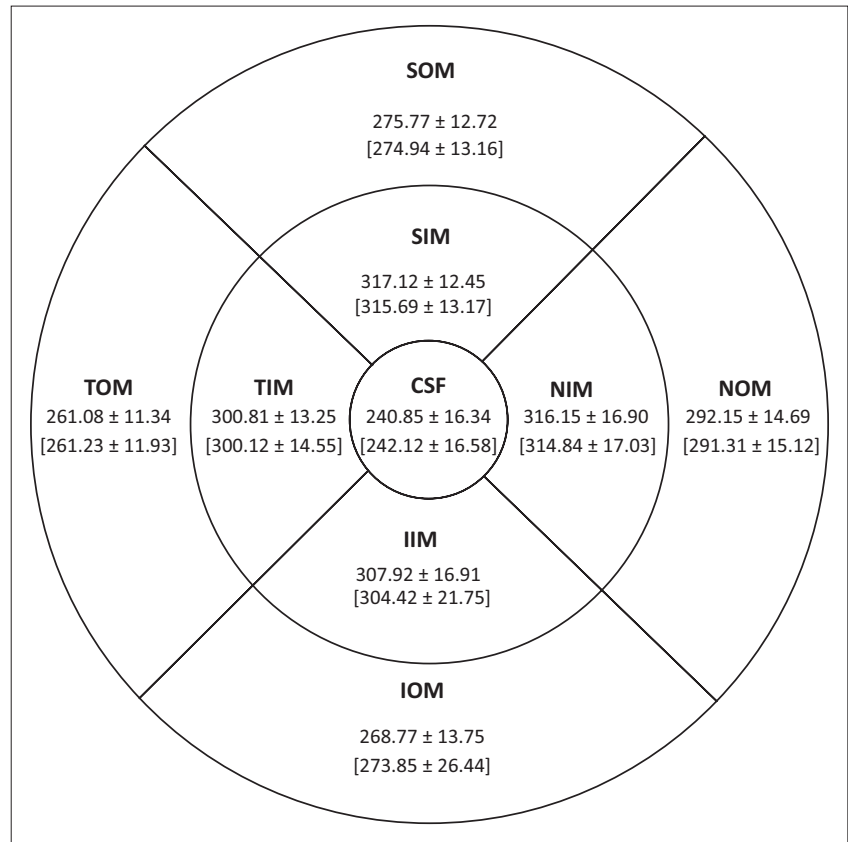

SOM, superior outer macular; SIM, superior inner macular; TOM, temporal outer macular; TIM, temporal inner macular; CSF, central subfield/fovea; NIM, nasal inner macular; NOM nasal outer macular; IIM, inferior inner macular; IOM, inner outer macular; ETDRS, Early Treatment Diabetic Retinopathy Study.

FIGURE 2: An Early Treatment Diabetic Retinopathy Study grid showing mean macular thicknesses and standard deviations (in $\mu \mathrm{m}$ ) for test and retest (square brackets) measurements for the right eyes of participants $(n=26)$.

TABLE 1: Probability values for Kolmogorov-Smirnov, Lilliefors and Shapiro-Wilk tests for test and retest retinal thickness measurements for the five main ETDRS regions.

\begin{tabular}{|c|c|c|c|c|c|}
\hline \multirow[t]{2}{*}{ Variable } & \multicolumn{2}{|c|}{ Kolmogorov-Smirnov } & \multirow[t]{2}{*}{ Lilliefors ( $p$ ) } & \multicolumn{2}{|c|}{ Shapiro-Wilk } \\
\hline & $d$ & $p$ & & $W$ & $P$ \\
\hline OCT CSF t & 0.0918 & $<$ n.s. & $<1.00$ & 0.9659 & 0.52 \\
\hline ОСТ CSF rt & 0.0926 & $<$ n.s. & $<1.00$ & 0.9753 & 0.76 \\
\hline ОСТ SIM t & 0.1059 & $<$ n.s. & $<1.00$ & 0.9502 & 0.24 \\
\hline ОСТ SIM rt & 0.1194 & $<$ n.s. & $<1.00$ & 0.9382 & 0.12 \\
\hline OCT NIM t & 0.1198 & $<$ n.s. & $<1.00$ & 0.9503 & 0.24 \\
\hline ОСT NIM rt & 0.1800 & $<$ n.s. & $<0.05$ & 0.9291 & 0.07 \\
\hline OCT TIM t & 0.1273 & $<$ n.s. & $<1.00$ & 0.9119 & 0.03 \\
\hline ОСт TIM rt & 0.1796 & $<$ n.s. & $<1.00$ & 0.9345 & 0.10 \\
\hline OCT IIM t & 0.1145 & $<$ n.s. & $<1.00$ & 0.9635 & 0.47 \\
\hline OCT ॥M rt & 0.1786 & $<$ n.s. & $<0.05$ & 0.9236 & 0.05 \\
\hline
\end{tabular}

Note: All significant values $(p<0.05)$ are indicated in bold text (i.e. deviation from normal distribution).

$d$, Kolmogorov-Smirnoff $\mathrm{D}$ statistic/value that results in a rejection of normally distributed data when it exceeds the critical value found within a statistical table; $p, p$-value; $\mathrm{t}$, test; $\mathrm{rt}$, retest; OCT, ocular coherence tomography; CSF, central subfield/fovea; SIM, superior inner macular; NIM, nasal inner macular; IIM, inferior inner macular; TIM, temporal inner macular ETDRS, Early Treatment Diabetic Retinopathy Study; n.s., non-significant.

Retinal thickness measurements in five regions including the CSF, SIM, NIM, IIM and TIM regions formed the focus of this study (see Figure 2).

The tests for normality of the data (Table 1) indicated that the majority of test and retest retinal thickness measurements were normally distributed. Even though parametric methods could be used to analyse the data, non-parametric methods were used, as the sample was small $(n=26)$. With the Shapiro-Wilk tests only one of the samples did not suggest normality (Table 1). If a Bonferroni correction was applied $(p<0.005)$ then none of the normality tests would be significant for the five ETDRS regions, implying that all retinal thickness measurements would be normally distributed.

\section{Oscillatory thresholds}

Figure 3 illustrates the pseudo-3D maps for Participant 1 (P1) and the ability of the VPS1 to rotate the map to view thresholds in their natural three-dimensional space. This rotation allows examiners to more easily assess or visualise magnitudes of the OT. Even though this feature is available and extremely useful, the majority of pseudo-3D maps herein will be rotated into the orientation seen in Figure 3 which is often better for explanation and understanding for examiners as well as easier for patient comprehension.

Figure 4 shows that OT is fairly similar between test and retest measurements, which was consistent for each participant in this study. The map with the smallest OT was found for P16 (test: $0.045 \mu \mathrm{m}$; retest: $0.056 \mu \mathrm{m}$ ), while the largest OT was found for P27 (test: $0.94 \mu \mathrm{m}$; retest: $0.98 \mu \mathrm{m}$ ). For the majority of participants, the pseudo-3D test and retest macular maps appeared similar to that of P20, with OT ranging from $0.11 \mu \mathrm{m}$ to $0.15 \mu \mathrm{m}$.

Using OS maps for all participants $(n=26)$, it was possible to use their thresholds to establish preliminary means and SDs at each of the nine points. Figures 5 and 6 indicate the overall test and retest means and SDs at each of the nine points for all right eyes. At the fovea, both test and retest mean OTs were $0.14 \mu \mathrm{m}$ (Figure 5). Figure 6 indicates that Point 8 in the retest had a slightly larger SD compared with the rest of the map, as well as the test map. When inspecting the macular OS field maps (MF 9) for the whole sample, an outlier was found for Point 8 (Participant 27) that caused the deviation.

Normality testing was also completed for the OT measurements. Table 2 shows that the OT samples were not normally distributed, indicating the need for non-parametric statistical methods to assess agreement, repeatability and reliability.

\section{Agreement and Bland-Altman plots}

There was good agreement between test and retest measurements of the CSF retinal thickness measurements and OT at the fovea (Point 5). Although the other BlandAltman plots were not included, it was evident that good agreement occurred between test and retest measurements for both retinal thickness and OT methods at the five specific regions and coordinates.

This is also evident in Tables 3 and 4 using the limits of agreement (LoA), standard errors (SE), coefficients of reliability $(C R)$, coefficients of variation $(C V)$ and intraclass coefficients (ICC), where mean differences $\left(\bar{X}_{\mathrm{d}}\right)$ were small (1.27 $\mu \mathrm{m}$ and $-0.006 \mu \mathrm{m}$ for retinal thickness and OT, respectively). The LoA were narrow $(22 \mu \mathrm{m}$ and $0.039 \mu \mathrm{m}$ for OCT and OT, respectively) with small SE. The CR 


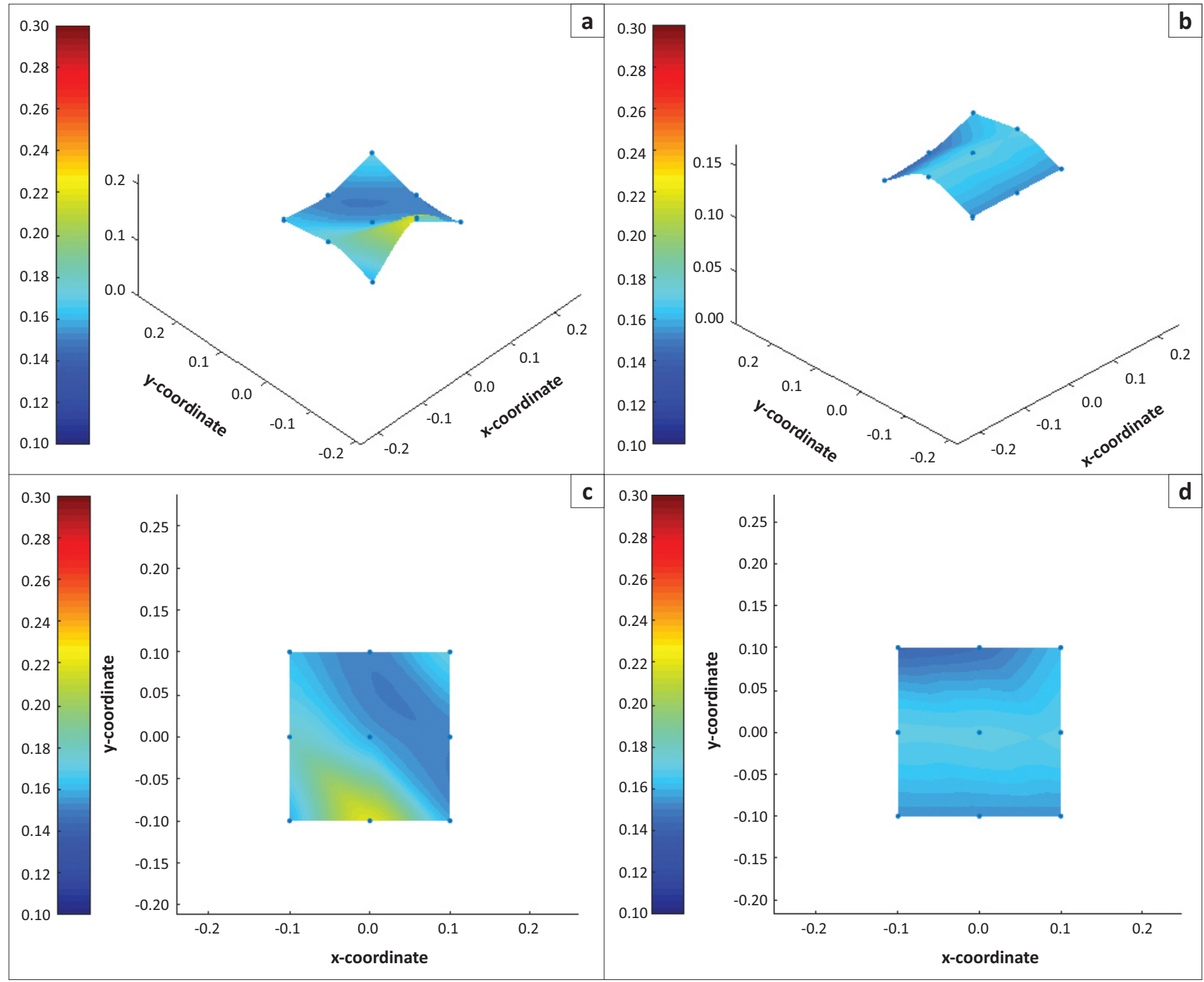

FIGURE 3: Pseudo-3D test $(\mathrm{t})$ and retest ( $\mathrm{rt}$ ) maps for the macular oscillatory threshold field test (MF 9) for Participant 1 (P1). As can be seen, the test and retest maps above illustrate the three-dimensional properties of the map (or surface), while the maps below are indicated in a frontal plane. Units for oscillatory thresholds are micrometres $(\mu \mathrm{m})$ but $x$ - and $y$-coordinates are in millimetres $(\mathrm{mm})$ on the monitor and not to scale. (a) P1 test, (b) P1 retest, (c) P1 test and (d) P1 retest.

values were small (7.83 and 0.02 were the smallest for retinal thickness and $\mathrm{OT}$, respectively); the $C V$ values were close to zero (the smallest being 0.04 and 0.04 for retinal thickness test and retest, respectively, as well as 0.08 and 0.09 for OT test and retest measurements, respectively). The ICC, which indicates the similarities between samples, showed that while retinal thickness test and retest measurements for each of the five regions were very similar, the OS test and retest measurements had slight variances (ICCs < 0.71) Overall, good test-retest agreement existed for the retinal thickness and OT measurements (Tables 3 and 4).

\section{Comparison of test and retest samples}

Matched pair tests, also known as Wilcoxon signed-rank tests, are based on the differences between test and retest measurements (usually retest minus test), which are then ranked along with the sign of the difference (that is, positive differences ranked together and negative differences ranked together) before being added to get the sum of the ranks (positive or negative). The test statistic $(T)$ is then the smaller of the two summed ranks. ${ }^{25}$ The results for the five main macular thickness regions and OS points using the matched pair test are presented in Tables 5 and 6 , respectively.

All the test and retest measurements for both retinal thickness and OS (with the exception of Point 5) were not significant at $p<0.05$. Using the Bonferroni correction the significance level changed to 0.002 and all comparisons yielded nonsignificant results. This means that measurements were reliable and repeatable.

\section{Correlation between optical coherence tomography and oscillatory sensitivity}

The correlation between macular thickness measurements and OT was investigated as it may be useful to use 


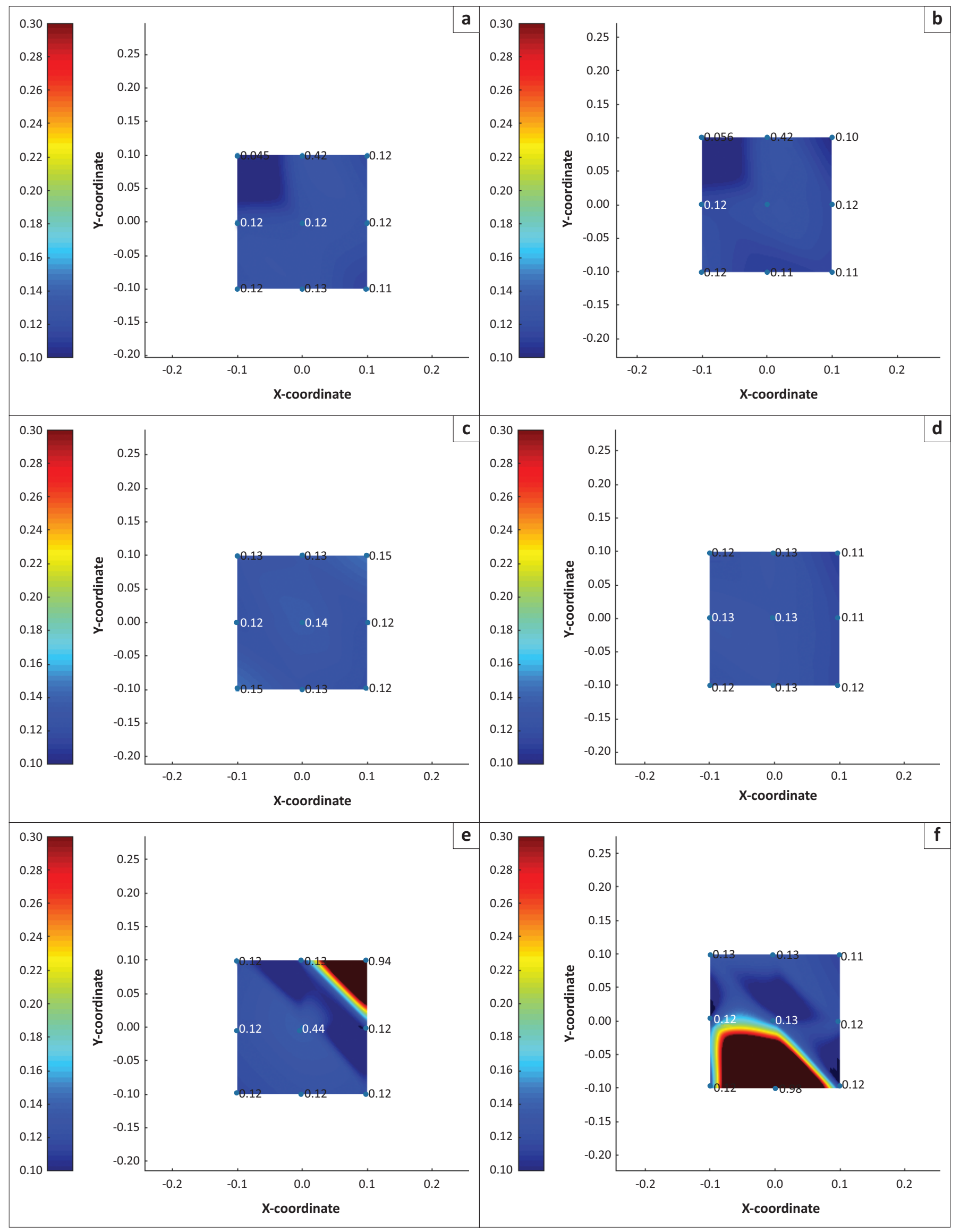

P, participant.

FIGURE 4: Pseudo-3D maps of oscillatory threshold for test $(\mathrm{t})$ and retest ( $\mathrm{rt}$ ) macular field tests for three participants $(\mathrm{P} 16, \mathrm{P} 20$ and $\mathrm{P} 27)$ chosen from the sample $(n=26)$ to illustrate eyes with the smallest, average (normal) and largest oscillatory thresholds. (a) P16 test, (b) P16 retest, (c) P20 test, (d) P20 retest, (e) P27 test and (f) P27 retest. 

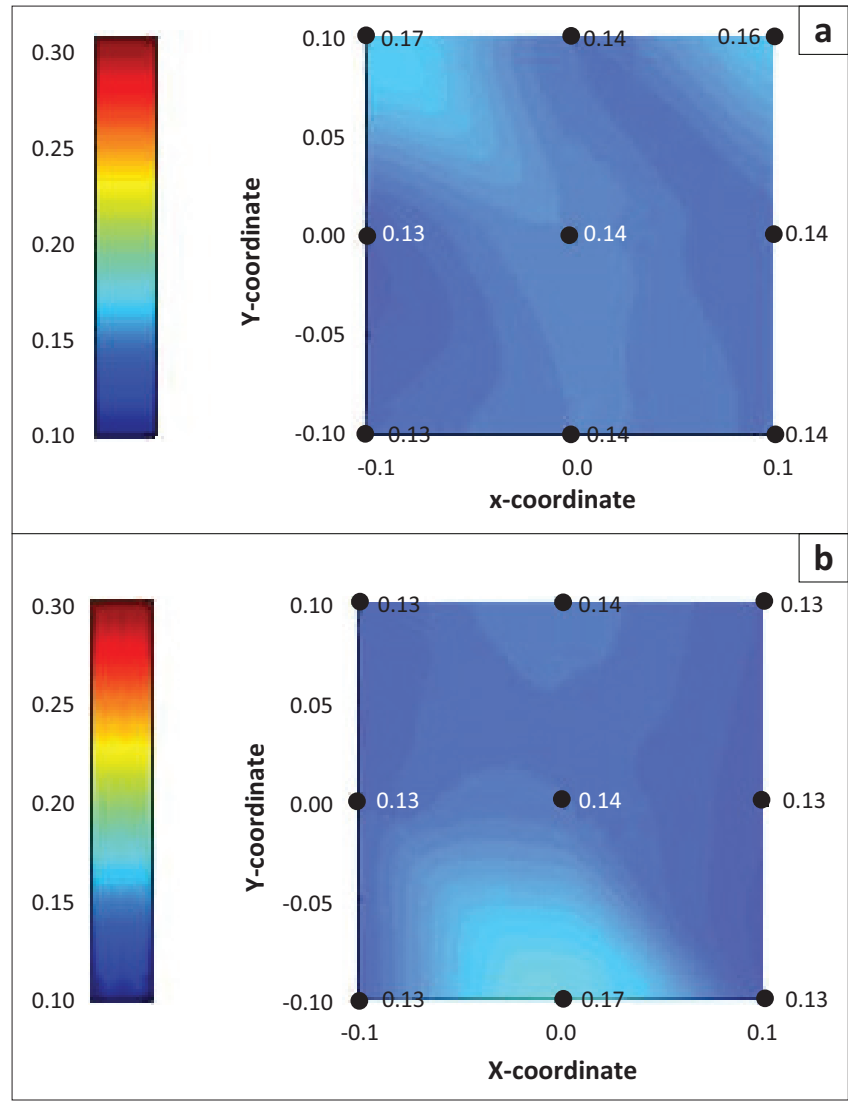

FIGURE 5: Pseudo-3D maps illustrating mean oscillatory threshold for (a) tes and $(b)$ retest measurements at each of the nine points within the macular field test for the sample $(n=26)$. (a) test and (b) retest.

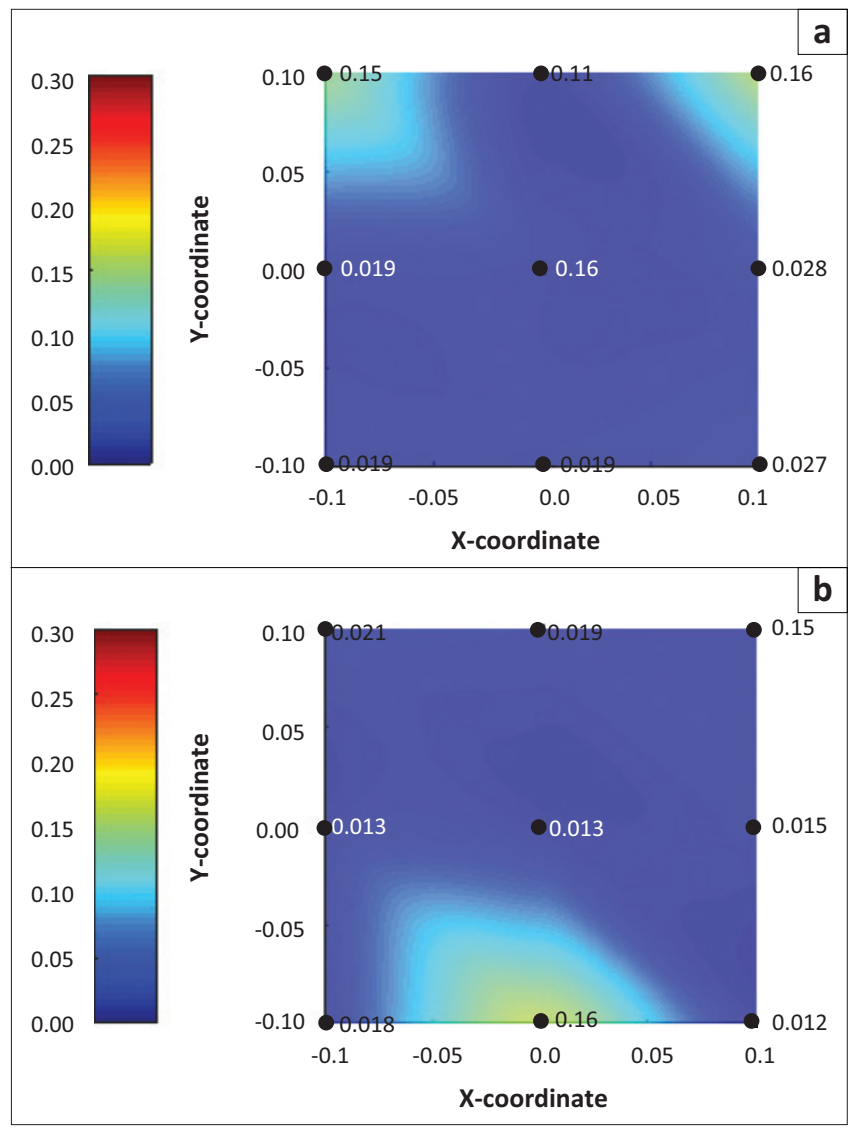

FIGURE 6: Pseudo-3D maps for standard deviations for oscillatory threshold for (a) test and (b) retest values at each of the nine points within the macular field test for the sample $(n=26)$ of right eyes. (a) test and (b) retest.
TABLE 2: Probability values for Kolmogorov-Smirnov, Lilliefors and Shapiro-Wilk tests for oscillatory sensitivity test and retest for the five main points investigated in the macular field test (MF 9).

\begin{tabular}{|c|c|c|c|c|c|}
\hline \multirow[t]{2}{*}{ Variable } & \multicolumn{2}{|c|}{ Kolmogorov-Smirnov } & \multirow{2}{*}{$\begin{array}{c}\text { Lilliefors } \\
p\end{array}$} & \multicolumn{2}{|c|}{ Shapiro-Wilk } \\
\hline & $d$ & $p$ & & $W$ & $p$ \\
\hline OS Point $5 \mathrm{t}$ & 0.1821 & $<$ n.s. & $<0.05$ & 0.8596 & $0.0005^{*}$ \\
\hline OS Point $5 \mathrm{rt}$ & 0.1935 & $<0.20$ & $<0.01$ & 0.8413 & $0.0000 *$ \\
\hline OS Point $2 t$ & 0.2072 & $<0.15$ & $<0.01$ & 0.8278 & 0.0261 \\
\hline OS Point $2 \mathrm{rt}$ & 0.2077 & $<0.15$ & $<0.01$ & 0.6575 & 0.0165 \\
\hline OS Point $4 \mathrm{t}$ & 0.1892 & $<$ n.s. & $<0.01$ & 0.9098 & $0.0022 *$ \\
\hline OS Point $4 \mathrm{rt}$ & 0.1471 & $<$ n.s. & $<0.10$ & 0.9010 & $0.0010 *$ \\
\hline OS Point $6 \mathrm{t}$ & 0.2471 & $<0.05$ & $<0.01$ & 0.5969 & $0.0000 *$ \\
\hline OS Point $6 \mathrm{rt}$ & 0.2435 & $<0.05$ & $<0.01$ & 0.7961 & $0.0002 *$ \\
\hline OS Point $8 \mathrm{t}$ & 0.2209 & $<0.10$ & $<0.01$ & 0.6886 & $0.0000 *$ \\
\hline OS Point $8 \mathrm{rt}$ & 0.4689 & $<0.01$ & $<0.01$ & 0.2635 & 0.0000 * \\
\hline
\end{tabular}

Note: Bold data represents significant results (i.e. deviation from normal distribution).

$d$, Kolmogorov-Smirnoff $\mathrm{D}$ statistic/value that results in a rejection of normally distributed data when it exceeds the critical value found within a statistical table; $p$, $p$-value; MF, macular field; $\mathrm{t}$, test; rt, retest; n.s., non-significant; $\mathrm{OS}$, oscillatory sensitivity.

*, Significant results when a Bonferroni correction was applied.
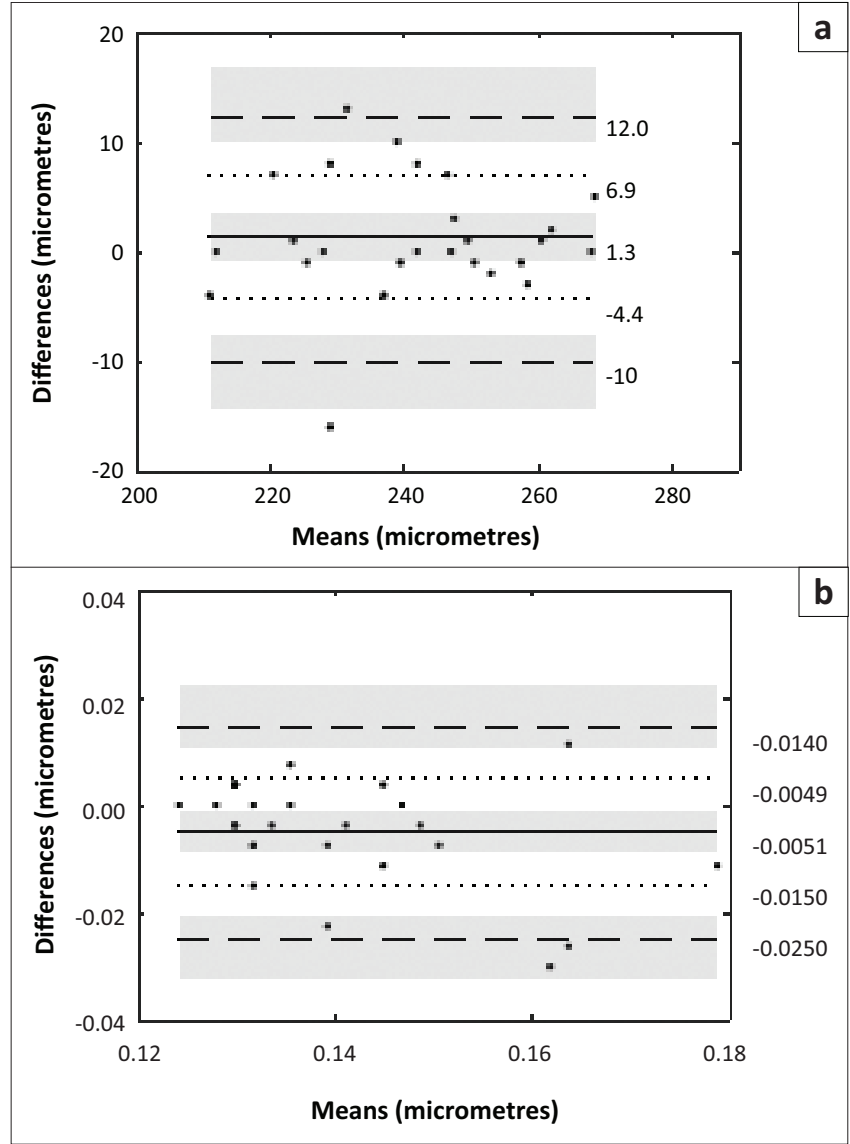

FIGURE 7: Bland-Altman plots of test and retest means versus differences for retinal thickness measurements at the fovea (central subfield/fovea) and oscillatory threshold at the fovea (point 5) for the sample $(n=26)$. (a) Bland-Altman thickness for central subfield/fovea $(r=0.043, p=0.83$ ) and (b) oscillatory threshold of Point 5 (central subfield/fovea) $(r=-0.38$, $p=0.05)$.

both methods clinically when evaluating certain ocular pathologies. Figure 8 indicates the correlation between macular thickness and OT at the fovea (central subfield/foveal thickness and Point 5). There was no relationship between OT and macular thickness $(r=-0.14$, $p=0.49$ and $r=0.03, p=0.89$ for test and retest samples, respectively). 
TABLE 3: Descriptive statistics for the Bland-Altman plots for retinal thickness in the central subfield/fovea, superior inner macular, nasal inner macular, inferior inner macular and temporal inner macular.

\begin{tabular}{|c|c|c|c|c|c|}
\hline Variable & CSF & SIM & NIM & $\mathrm{IIM}$ & TIM \\
\hline$\overline{\bar{x}}(S D)$ & $241.48(16.22)$ & $316.40(12.68)$ & $315.500(16.680)$ & 306.17 (18.79) & $300.46(13.70)$ \\
\hline $\bar{X}_{d}(S D)$ & $1.27(5.65)$ & $-1.42(3.72)$ & $-1.310(6.200)$ & $-3.50(10.29)$ & $-0.69(4.89)$ \\
\hline$S E$ for $\bar{X}_{d}$ & 1.11 & 0.73 & 1.220 & 2.02 & 0.96 \\
\hline (LLoA; ULoA) & $(-10.04 ; 12.35)$ & $(-8.87 ; 5.87)$ & $(-13.710 ; 10.850)$ & $(-24.09 ; 16.67)$ & $(-10.47 ; 8.89)$ \\
\hline SE for LoA & 1.92 & 1.26 & 2.110 & 3.50 & 1.66 \\
\hline$C R$ & 11.37 & 7.83 & 12.430 & 21.35 & 9.68 \\
\hline$C V_{\text {retest }}$ & 0.07 & 0.04 & 0.054 & 0.07 & 0.05 \\
\hline ICC & 0.94 & 0.95 & 0.930 & 0.84 & 0.94 \\
\hline
\end{tabular}

Note: All units are in micrometres except $C R, C V$ and ICC.

CSF, central subfield/fovea; SIM, superior inner macular; NIM, nasal inner macular; IIM, inferior inner macular; TIM, temporal inner macular; SD, standard deviation; SE, standard error; LLoA, lower limit of agreement ; ULOA, upper limit of agreement; LoA, limit of agreement; $C R$, coefficients of reliability; CV, coefficients of variation; ICC, intraclass coefficient.

TABLE 4: Descriptive statistics for the Bland-Altman plots for oscillatory sensitivity of Points 2, 4, 5 (fovea), 6 and 8.

\begin{tabular}{lcccc}
\hline Variable & Point 5 & Point 2 & Point 4 & Point 6 \\
\hline $\bar{X}(S D)$ & $0.140(0.014)$ & $0.1390(0.0130)$ & $0.1320(0.0150)$ & $0.1326(0.0190)$ \\
$\bar{X}_{d}(S D)$ & $-0.006(0.010)$ & $0.0015(0.0140)$ & $0.0010(0.0150)$ & $-0.0060(0.0220)$ \\
$S E$ for $\bar{X}_{d}$ & 0.002 & 0.0030 & 0.0030 & $0.1570(0.0817)$ \\
(LLOA; ULOA) & $(-0.025 ; 0.014)$ & $(-0.0270 ; 0.0290)$ & $(-0.0290 ; 0.0310)$ & $(-0.0490 ; 0.0370)$ \\
$S E$ for LOA & 0.003 & 0.0048 & 0.0051 & 0.0040 \\
$C R$ & 0.020 & 0.0300 & 0.0300 & 0.0074 \\
$C V_{\text {test }}$ & 0.110 & 0.0800 & 0.1500 & 0.0500 \\
$C V_{\text {retest }}$ & 0.090 & 0.1300 & 0.1000 & 0.2000 \\
$I C C$ & 0.710 & 0.5700 & 0.5900 & 0.050 \\
\hline
\end{tabular}

Note: All units are in micrometres except for $C R, C V$ and $I C C$. Bold data signify outliers.

$S D$, standard deviation; SE, standard error; LLoA, lower limit of agreement ; ULOA, upper limit of agreement; LoA, limit of agreement; $C R$, coefficients of reliability; $C V$, coefficients of variation; ICC, intraclass coefficient.

TABLE 5: The Wilcoxon matched pair hypothesis tests comparing test and retest measurements for the retinal thicknesses measurements within the macular region.

\begin{tabular}{lccc}
\hline Variable & $\boldsymbol{T}$ & $\boldsymbol{Z}$ & $\boldsymbol{p}$-level \\
\hline OCT CSFt and OCT CSFrt & 81.00 & 1.199 & 0.23 \\
OCT SIMt and OCT SIMrt & 94.50 & 1.586 & 0.11 \\
OCT NIMt and OCT NIMrt & 77.00 & 1.338 & 0.18 \\
OCT IIMt and OCT IIMrt & 110.50 & 0.836 & 0.40 \\
OCT TIMt and OCT TIMrt & 70.50 & 0.653 & 0.51 \\
\hline
\end{tabular}

$T, T$-value is a test statistic calculated using the sample data and comparing data to expected null hypothesis; $Z$, number of standard deviations from the mean; OCT, ocular coherence tomography; CSF, central subfield/fovea; SIM, superior inner macular; NIM, nasal inner macular; IIM, inferior inner macular; TIM, temporal inner macular; $t$, test; rt, retest.
TABLE 6: The Wilcoxon matched pair hypothesis tests comparing test and retest measurements for oscillatory thresholds at Points 2, 4, 5, 6 and 8 .

\begin{tabular}{lccc}
\hline Variable & $\boldsymbol{T}$ & $\boldsymbol{Z}$ & $\boldsymbol{p}$-level \\
\hline Point $5 \mathrm{t}$ and Point 5 rt & $\mathbf{5 5 . 5}$ & $\mathbf{2 . 0 8 5}$ & $\mathbf{0 . 0 4}$ \\
Point 2 t and Point 2 rt & 99.0 & 0.224 & 0.82 \\
Point 4 t and Point 4 rt & 132.0 & 0.182 & 0.86 \\
Point 6 t and Point 6 rt & 80.0 & 1.509 & 0.13 \\
Point 8 t and Point 8 rt & 84.0 & 1.095 & 0.27 \\
\hline
\end{tabular}

Note: Bold data indicate significant values (where $p<0.05$ )

$\mathrm{t}$, test; rt, retest; $T, T$-value is a test statistic calculated using the sample data and comparing data to expected null hypothesis; $Z$, number of standard deviations from the mean.

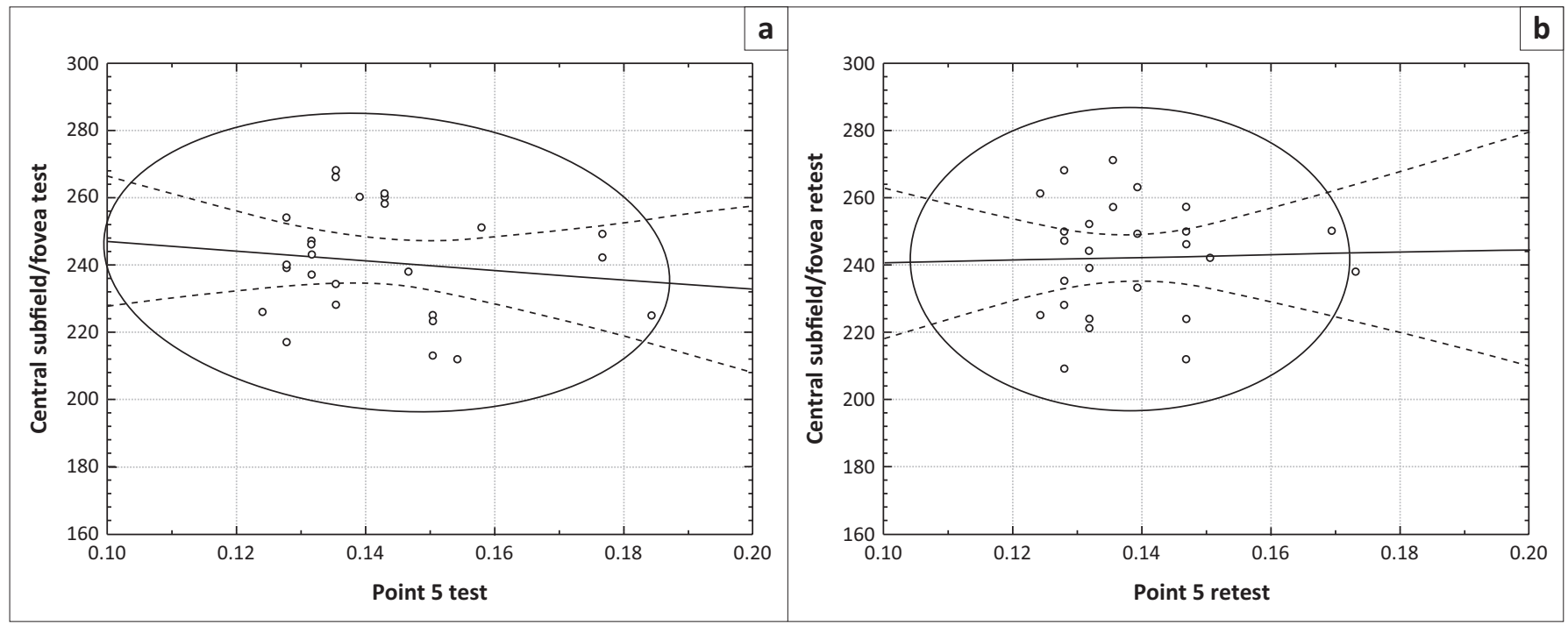

FIGURE 8: Scatter plots for (a) test and (b) retest measurements of oscillatory threshold $(\mu \mathrm{m})$ versus ocular coherence tomography $(\mu \mathrm{m})$ for the sample $(n=26)$. 


\section{Discussion}

For retinal thickness measurements, good reliability and repeatability occurred when using the iVue 100 OCT instrument. This was shown in the small differences between test and retest thickness measurements (Figure 2), the good agreement when analysing the data with the Bland-Altman plots (Figure 7 and Table 3) and hypothesis tests, which indicated that test and retest measurements were similar and repeatable (Table 5). Similarly, good repeatability was found for test and retest OS measurements as initial OT means and SDs were similar (Figures 5 and 6), the agreement was good using Bland-Altman plots (Figure 7 and Table 4) and hypothesis tests (using Bonferroni correction) resulted in repeatable measurements.

Thus, test and retest samples for retinal thickness measurements using iVue 100 OCT instrument and visual sensitivity using OS indicated that these are reliable methods.

Limitations of this study included the relatively small, conveniently selected sample, where factors such as ethnicities were not controlled. There were also occasional input errors resulting in duplicated or missed points, which had the effect of decreasing the sample size. It is therefore recommended that future studies focus more on establishing normative macular thickness and OT data for all ethnicities, ages and genders along with establishing ways to reduce input errors. It may also be beneficial to assess if differences exist between eyes (left vs. right) or to examine different types and magnitudes of refractive errors as well as systemic and different ocular pathologies. Correlation studies between OT for diseased eyes and macular thickness using the iVue 100 OCT should also be further investigated as it is postulated that the relationship may be different in diseased eyes (as against the young healthy eyes in this article). In spite of the limitations in this study, it was possible to establish that macular thickness and OT resulted in reliable and repeatable test-retest measurements. Consequently, the VPS1 and iVue 100 OCT instruments can be used with confidence in future in both clinical and research settings. This research was also the first to establish test and retest repeatability of the VPS1 program for central field (CF 49), macular field (MF 9) and foveal meridional discrimination $\left(\mathrm{FMD} 15^{\circ}\right)$, with the focus here mainly on macular measurements.

\section{Conclusion}

Oscillatory sensitivity and OT are new concepts and therefore it was necessary to investigate the reliability of test and retest measurements before larger, non-random samples could be used to determine normative data. Although a relatively small sample was used in this study, it is evident that OS and OT can be used in further studies and to clinically assess vision function. The good repeatability of the macular thickness measurements with the iVue 100 OCT instrument indicates that it can also be used in comparison with other OCT instruments in future studies and may be used with confidence clinically in measuring retinal thicknesses.

\section{Acknowledgements Competing interests}

The authors declare that they have no financial or personal relationships that may have inappropriately influenced them in writing this article.

\section{Authors' contributions}

A.H. collected the data and wrote the manuscript as part of her master's thesis under the supervision of A.R. Review of the initial draft, supervision of revisions and approval of the manuscript were performed by A.R.

\section{References}

1. Atwood E, Ercolano V, Erkess E, Searing, L, editors. Stedman's medical dictionary. 28th ed. Baltimore, MD: Lippincott Williams \& Wilkins; 2006.

2. Humphreys GW, Riddoch MJ, Boucart M. The breakdown approach to visual perception: Neuropsychological studies of object recognition. In: Humphreys GW editor. Reading in mind and language: Understanding vision. MA: Blackwel Publishers; 1992; p. 104-125.

3. Coon D, Mitterer JO. Introduction to psychology: Active learning through modules. 12th ed. China: Wadsworth Cengage Learning; 2012; p. 150-203.

4. Goldstein EB. Cognitive psychology. 3rd ed. China: Wadsworth Cengage Learning; 2011.

5. Matthews N, Qian N. Axis-of-motion affects direction discrimination, not speed discrimination. Vision Res. 1999;39:2205-2211. https://doi.org/10.1016/S0042 6989(98)00300-9

6. Georgeson MA, Scott-Samuel NE. Spatial resolution and receptive field height of motion sensors in human vision. Vision Res. 2000;40:745-758. https://doi. org/10.1016/S0042-6989(99)00219-9

7. Hanada M. Investigation of center-surround interaction in motion with reaction time for direction discrimination. Vision Res. 2012;59:34-44. https://doi. org/10.1016/j.visres.2012.02.009

8. Silva $A E$, Liu Z . Opponent backgrounds reduce discrimination sensitivity to competing motions: Effects of different vertical motions on horizontal motion perception. Vision Res. 2015;113:55-64. https://doi.org/10.1016/j.visres.2015. 05.007

9. Fujimoto JG. Optical and acoustical imaging of biological media: Optical coherence tomography. Comptes Rendus. 2001;4:1099-1111.

10. Schwartz SH. Visual perception: A clinical orientation. 4th ed. New York: McGrawHill; 2010.

11. Rebolleda G, Diez-Alvarez L, Casado A, et al. OCT: New perspectives in neuroophthalmology. Saudi J Ophthalmol. 2015;29:9-25. https://doi.org/10.1016/j. sjopt.2014.09.016

12. Fercher AF. Optical coherence tomography. J Biomedical Optics. 1996;2:157-173. https://doi.org/10.1117/12.231361

13. Broecker EH, Dunbar MT. Optical coherence tomography: Its clinical use for the diagnosis, pathogenesis, and management of ocular conditions. Optometry. 2005;76:79-101. https://doi.org/10.1016/S1529-1839(05)70262-1

14. Mashige KP, Oduntan OA. Macular thickness and their associations with ocular and demographic variables in black South Africans. Afr Vision Eye Health. 2017;76:1-7.

15. Murugan C, Golodza BZ, Pillay K, et al. Retinal thickness in Black and Indian myopic students at the University of Kwazulu-Natal. Afr Vision Eye Health. 2015;74:1-7. https://doi.org/10.4102/aveh.v74i1.300

16. Han IC, Jaffe GJ. Comparison of spectral and time domain optical coherence tomography for retinal thickness measurements in healthy and diseased eyes. Am J Ophthalmol. 2009;147:847-858. https://doi.org/10.1016/j.ajo. 2008.11.019

17. Huang J, Liu X, Wu Z, et al. Macular thickness measurements in normal eyes with time domain and Fourier domain optical coherence tomography. Retina. 2009;29:980-987. https://doi.org/10.1097/IAE.0b013e3181a2c1a7

18. Kiernan DF, Hariprasad SM, Chin EK, et al. Prospective comparison of Cirrus and Stratus optical coherence tomography for quantifying retinal thickness. Am J Ophthalmol. 2009;147:267-275. https://doi.org/10.1016/j.ajo.2008.08.018

19. Wolf-Scnurrbusch UEK, Ceklic L, Brinkmann CK, et al. Macular thickness measurements in healthy eyes using six different optical coherence tomography instruments. Invest Ophthalmol Vis Sci. 2009;50:3432-3437. https://doi. org/10.1167/iovs.08-2970 
20. Bafiq R, Mathew R, Pearce E, et al. Age, sex and ethnic variations in inner and outer retinal and choroidal thickness on spectral-domain optical coherence tomography. Am J Ophthalmol. 2015;160:1034-1043. https://doi.org/10.1016/j.ajo.2015.07.027

21. Budenz DL, Anderson DR, Varma R, et al. Determinants of normal retinal nerve fibre thickness measured by Stratus OCT. Ophthalmology. 2007;114:1046-1052. https://doi.org/10.1016/j.ophtha.2006.08.046

22. Razali NM, Wah YB. Power comparisons of Shapiro-Wilk, Kolmogorov-Smirnov, Lilliefors and Anderson-Darling tests. J Stat Model Analytics. 2011;2:21-33.
23. Hasrod NG. Colour discrimination and the Cambridge colour test: Reliability of discrimination ellipses and trivectors in colour space [dissertation]. Johannesburg: University of Johannesburg, South Africa; 2016.

24. Grey C. Kolmogorov-Smirnov and Lilliefors test [homepage on the Internet]. University of Minnesota; 2013. [cited 2017 Apr 11]. Available from: http://www. stat.umn.edu/geyer/5601/examp/kolmogorov.htm

25. Field A. Discovery statistics using SPSS. 3rd ed. London: Sage; 2011. 